\title{
Study on the Importance of Ground Training in Dance Basic Skills
}

\author{
Nuowa $\mathrm{Wu}$ \\ Anshan Normal College \\ Anshan, China
}

\begin{abstract}
In the process of professional training for dancers, ground training helps dancers to achieve a beautiful body shape, elegant temperament, improve their professional ability and body shape. Relatively speaking, the Chinese classical dance requires relatively higher basic skills, the body flexibility of the dancers are more demanding, while the ground training is mainly the training of upper and lower limbs soft opening and flexibility to improve the dance learner from top to bottom soft opening, thereby increasing the joints of the scope of activities and muscles, ligaments, tendons and other organizations, the ability to stretch and shrink, so that the body's flexibility have been balanced development .All in all, after a long period of ground training, it can change the habit of habitual relaxation of their own dancers, training a good professional body.
\end{abstract} breath

Keywords-Ground training; yoga: flexibility; flexibility;

\section{INTRODUCTION}

Ground training is basic training course to improve and strengthen the scientific and systematic training. To achieve effectiveness, ground training plays an important role in cultivating students' physical skills, body language ability and becoming outstanding dance artists in professional teaching and training courses. To some extent, ground training contains a certain mandatory. If it is not mandatory, it cannot change the normal body habit up to the superhuman flexibility and strength. In such training, conflicts are inevitable. Well-handled can speed up the training process. Training correct and good sense of movement and dance awareness, to support the construction and completion of the middle of the action to provide capacity and support, so ground training is very important and it is the basis for the basic skills of Chinese classical dance, it is the thing that every dancers should focus!

\section{GROUND TRAINING IS AN INTEGRAL PART OF DANCE TRAINING}

Ground training is one of the basic forms of dance training, in classical dance, if one didn't have a good basic skill, one can't dance a great classical dance and ground training is mainly directed at the soft body of the dancers, strength, coordination of the opening. The training course laid a very important foundation for the dancers to practice their basic skills. The requirements of dance for one's soft opening, control, coordination and stability are quite strict. So in order to dance well, you must practice your basic skills well. Therefore, ground training plays an important role in the quality of basic skills. Through further training on the ground, dancers can master normative and solid basic skills and technical skills on the basis of their own to achieve the unity of body and manner. Due to the characteristics of dance art, all dancers are required to be upright in their basic stance, and their movements should be diversified. Exercise on the ground can achieve relative tension and relative balance of anti-muscle groups. After a long period of ground practice, it can solve the natural relaxation of the habit of dancers and train out a good posture. And it can initially grasp the tension and relaxation of their own muscles, exercise back and leg ability to solve the waist and leg softness and control ability. Ground training can strengthen the body's flexibility and strength, strengthen the feeling of muscle strength and movement, and improve the ability of limb control and stability of the center of gravity. Ground training has a direct impact on attitude and form. Good flexibility and strength of quality is the foundation of completing the technical actions and improving the quality of movement. As the difficulty of training increases, its jumping, balancing and liableness need flexibility and strength's achievement. The ground training position is the most fundamental and critical for basic training.

\section{THE CONTENT OF GROUND TRAINING}

Ground training's content, mainly can be summarized as flexible training (commonly known as soft-opening training), strength training (also known as ability training), technology, skills, action training .Flexibility, strength and technical skills are the three major technical components that make up the dancer's career. It is also the basic physical and technical condition that a professional dancer must possess and achieve. The main content of ground training is divided into four aspects:

- Body Flexibility: In terms of magnitude of human activity. The more flexible the dancer, the dancing posture will be more elegant and beautiful.

- Muscular Strength: the strength that the dancers need in their dance process is muscle power, control and jumping power. They are primarily an ability that occurs when muscle contracts or tension increases. 
- Control And Stability of Body: control refers to the muscles in the dance tension to maintain balance control and to ensure the formation of a fixed force dancing; stability refers to the performance of the adjustment, control, restore the body's ability to balance and stability.

- Coordination and Flexibility of Body: Coordination refers to the body muscles that can coordinate with each other; flexibility refers to the body or limb that can quickly change the position and direction of certain aspects of the ability.

One of the purposes of ground exercises is to overcome the natural form and master the dance skills. In fact, any dance needs basic skills, whether it is national dance, Latin dance, modern dance and hip-hop, all of them require basic skills. When the basic skills are good, many difficult movements can be done. It can be said that practicing basic skills is the basis for dancing well. Moreover, ground training can also provide a solid foundation for dancers to practice basic skills.

\section{THE WAY OF GROUND TRAINING}

Ground training generally focus on solving the waist and legs' softness and shoulder, hip substantial training and the ability to waist, leg work and toes straight open capacity. Dance of the "open, jump, straight" these three elements have the function of contracting muscle fibers, the movement in the combination of static and dynamic consumption of excess fat, yoga is from India's ancient fitness, which means "join, unite, combine and bondage" , which can be extended to "combination", "connection." The essence of yoga is to combine the spirit and the flesh into the best condition, combine life and the universe into the perfect state, and harmonize the inner peace with the health of the body to achieve the unity of the mind and the heart. After training, people will become more slender. Therefore, although there are physical exertions, they are also exercise and its action is more emphasis on muscle endurance, body flexibility, exercise intensity which is not too difficult and most people can accept. We can use the combination of yoga and dance ground exercises for basic training, but the most important thing is to solve the soft opening of the dancers' legs and feet, some dancers has a good soft opening ability, but some dancers just the opposite. Thus to overcome its own natural form, it is necessary to ensure the quantity and quality of practice; on this basis, in order to increase the performance of dancers, dancers must do a variety of combination exercises. Therefore, it is necessary for them to overcome the initial problems and master the correct form through systematic ground training; Training the flexibility of their limbs and forming the tension and relaxation of control ability of their respective muscles and the flexibility of the joints, they should do a good job of ground training in both static and dynamic exercises in two ways:

- Static Exercise: It is through the slow dynamic stretching, the muscles, tendons, ligaments and other soft tissue elongated, and stay for a certain period of practice methods, such as consumption of legs, waist, moving legs. Static tension according to different requirements can be active and passive stretching method. Active static exercise refers to the maximum range of motion, with its own muscle strength to maintain the state of motionless, such as Su Yan, explore the sea control dance practice; passive static exercise is to use external force to maintain a fixed position, such as a dancer to help another dancer's hips slowly while pressing hip exercises. Static stretching requires dancers feel sour, swell, pain, and to maintain a certain period of time, about 10 seconds.

- Power Exercise: It refers to the rhythmic, faster, gradually increasing the amplitude, repeated several times a stretch of action, such as the waist, waist, continuous kicking practice.

The above two methods are compared, the static tensile has the following characteristics:

First, good tensile strength control can prevent the occurrence of damage; Moreover, the training process of human energy consumption is small; Through the ground training to a certain extent, muscle capacity can be enhanced. Therefore, a vigorous, fast paced, bold, daring choreography not only requires strength, but also "speed strength", muscular endurance, control and jumping power. A poorly flexible dancer, the action is difficult to be quiet, gentle; if the leg lift is not high, waist do not go, and the action will certainly look awkward and stiff.

\section{THE IMPORTANCE OF GROUND TRAINING ON THE DANCE}

\section{A. It is conducive to strengthening the body's flexibility and strength of quality}

Ground training has high strength requirements for the dancer's flexibility. It has a direct impact on attitude and form. Good flexibility and strength of quality is to complete the technical actions and improve the quality of the foundation. As the difficulty of basic training increases, its jumping, balancing and liableness need flexibility and strength to complete. It is also extremely important for professional training to take its place.

1) Flexibility: Flexibility is good or bad by the body's various joints in the movement of the opening width of the size of the decision. It has a congenital and acquired, congenital refers to the bone structure. Everyone has their own different bone structure, this is from a genetic point of view, is qualitative, in the process of training on the ground this is not easy to change. Ground training is accomplished by the inherent resistance of muscles. The basic training contents of ground training are open, stretch, straight and vertical. It has the function of improving body flexibility and contracting muscle fibers, and makes the body parts develop evenly. During the training process, the flexibility, strength and balance of the learner's leg and torso can be effectively developed. With the help of the body's slowmotion and decomposition exercises, the flexibility and 
flexion-and-extension capacity of the hip, knee and ankle can be improved.

2) Power: The strength requirement for professional ground training is the unnatural muscle shape that a learner's body or part of a body reacts violently. It has the effect of rapidly shrinking, lifting or tense the muscles. When conducting ground training, the most basic requirement is to open, stretch, straight, stand. One of the tension, straightness and strength training is the core of the core, so in the training process by rubbing, squatting, kicking, half toes and other exercises we should enhance leg and knee support strength and lumbar abdomen and the power of muscle contraction. So people can complete their action steady.

\section{B. It is conducive to strengthening the body shape}

Training on the ground has an important role for dancers' good shape, after a long time correct and standardized practice, can often stimulate the muscles of the leg muscles ,chest and back so that the dancer's legs, buttocks muscles on the lower limbs elongated center of gravity, so that the legs and buttocks have beautiful lines. As well as increase the chest and back muscle lines sense of elongation, so that dancers will be tall and straight. So it can leads to a better shape.

\section{It is conducive to training the correctness of the action}

Ground training is conducive to cultivate the ability of dancers to judge the reaction, create a beautiful body posture, and improve the performance of the United States, rhythmic beauty. A variety of elegant static posture and a variety of agile coordination of dynamic action can quickly respond and accurate through a single action and complete sets of actions to achieve gestures standardization.

\section{CONCLUSION}

In summary, we can see ground training has an important significance in dance. In the process it has a tremendous role for dancers to change the physical form, improve physical fitness and strengthen dance training. No matter what kind of dancing dancers show, what style of dance performance, every dancer must have a professional basis and ground training will lay a solid foundation for them.

\section{REFERENCES}

[1] Yuanmin Shen. Chinese Classical Dance Basic Training Course, Shanghai Music Publishing House.

[2] Peiying Wang. Basic Skills of Chinese Classical Dance Tutorial, Shanghai Music Publishing House.

[3] Jiantai Xiong. Basic Training Materials and Teaching Method of Chinese Classical Dance, Shanghai Music Publishing House.

[4] Yuanmin Shen. Chinese Classical Dance Training Methods, Shanghai music Publishing House. 